\title{
Linking Pain and the Body: Neural Correlates of Visually Induced Analgesia
}

\author{
Matthew R. Longo, ${ }^{1,2}$ Gian Domenico Iannetti, ${ }^{3}$ Flavia Mancini, ${ }^{2,4}$ Jon Driver, ${ }^{2}$ and Patrick Haggard ${ }^{2}$ \\ ${ }^{1}$ Department of Psychological Sciences, Birkbeck, University of London, London WC1E 7 HX, United Kingdom, ${ }^{2}$ Institute of Cognitive Neuroscience, \\ University College London, London WC1N 3AR, United Kingdom, ${ }^{3}$ Department of Neuroscience, Physiology and Pharmacology, University College \\ London, London WC1E 6BT, United Kingdom, and ${ }^{4}$ Department of Psychology, University of Milano-Bicocca, 20126, Milan, Italy
}

The visual context of seeing the body can reduce the experience of acute pain, producing a multisensory analgesia. Here we investigated the neural correlates of this "visually induced analgesia" using fMRI. We induced acute pain with an infrared laser while human participants looked either at their stimulated right hand or at another object. Behavioral results confirmed the expected analgesic effect of seeing the body, while fMRI results revealed an associated reduction of laser-induced activity in ipsilateral primary somatosensory cortex (SI) and contralateral operculoinsular cortex during the visual context of seeing the body. We further identified two known cortical networks activated by sensory stimulation: (1) a set of brain areas consistently activated by painful stimuli (the so-called "pain matrix"), and (2) an extensive set of posterior brain areas activated by the visual perception of the body ("visual body network"). Connectivity analyses via psychophysiological interactions revealed that the visual context of seeing the body increased effective connectivity (i.e., functional coupling) between posterior parietal nodes of the visual body network and the purported pain matrix. Increased connectivity with these posterior parietal nodes was seen for several pain-related regions, including somatosensory area SII, anterior and posterior insula, and anterior cingulate cortex. These findings suggest that visually induced analgesia does not involve an overall reduction of the cortical response elicited by laser stimulation, but is consequent to the interplay between the brain's pain network and a posterior network for body perception, resulting in modulation of the experience of pain.

\section{Introduction}

The experience of our own body is fundamentally multisensory. Parietal and premotor associative areas combine visual and somatosensory signals, providing multisensory representations of the body and peripersonal space (Graziano et al., 1994). Several studies show interplay between vision and somatosensation, including links in spatial attention (Kennett et al., 2001a), integration of cross-modal inputs by parietal neurons (Duhamel et al., 1998), and modulation of tactile acuity by vision of the body (Kennett et al., 2001b). Less is known about effects of multisensory signals on pain.

We recently discovered that viewing the body reduces acute pain. Participants rated nociceptive laser stimuli as less painful when viewing the stimulated hand in a mirror-box, versus an object at the same location (Longo et al., 2009). We subsequently replicated this effect using contact heat pain thresholds (Mancini et al., 2011), but the brain mechanisms underlying this "visually

\footnotetext{
Received Aug. 5, 2011; revised Nov. 23, 2011; accepted Dec. 20, 2011.

Author contributions: M.R.L., G.D.I., J.D., and P.H. designed research; M.R.L., G.D.I., F.M., and P.H. performed research; M.R.L. analyzed data; M.R.L., G.D.I., F.M., J.D., and P.H. wrote the paper.

M.R.L. was supported by a Biotechnology and Biological Sciences Research Council project grant (BB/D009529/1) to P.H. P.H. was further supported by a Leverhulme Trust Research Fellowship, and by EU FP7 Integrated Project VERE. G.D.I. is supported by a University Research Fellowship of The Royal Society. J.D. is a Royal Society Anniversary Professor. F.M. was funded by the doctoral program of the University of Milano-Bicocca.

The authors declare no competing financial interests.

Correspondence should be addressed to Matthew R. Longo, Department of Psychological Sciences, Birkbeck, University of London, Malet Street, London WC1E 7 HX, UK. E-mail: m.longo@bbk.ac.uk.

DOI:10.1523/JNEUROSCI.4031-11.2012

Copyright $\odot 2012$ the authors $\quad 0270-6474 / 12 / 322601-07 \$ 15.00 / 0$
}

induced analgesia" are unknown. Since the location and timing of stimulation were similar across conditions, attentional explanations appear unlikely. Instead, visual analgesia might reflect interplay between networks involved in viewing the body, and networks responsive to nociceptive inputs.

A putative "pain matrix" has been identified as a set of brain regions activated by nociceptive inputs (Tracey and Mantyh, 2007; Iannetti and Mouraux, 2010), including brainstem and thalamic nuclei, somatosensory areas SI and SII, insular, and anterior cingulate cortices. While activity in these areas scales parametrically with pain intensity (Derbyshire et al., 1997; Iannetti et al., 2005a), recent studies suggest that little pain matrix activity is nociceptive-specific. Rather, similar activations can be produced by tactile, auditory, and visual stimuli (Mouraux and Iannetti, 2009; Mouraux et al., 2011). The multisensory nature of this network makes it a likely candidate for cross-modal modulation of pain.

Extensive areas of posterior cortex are involved in perceiving and representing the human body. Neuroimaging studies reveal areas of occipital-temporal cortex preferentially responsive to seen bodies and body parts, including the extrastriate body area (EBA; Downing et al., 2001), and fusiform body area (Peelen and Downing, 2005). One recent study found an extensive topographic map of viewed body parts throughout occipitotemporal cortex (Orlov et al., 2010). A different form of body representation exists in the superior parietal lobule, where individual neurons respond to combinations of visual, tactile and auditory inputs near the body (Duhamel et al., 1998). Representations of 
our "body image" have been localized to the posterior parietal cortex (Critchley, 1953; Longo et al., 2010).

The two networks related to the experience of pain and to vision of the body seem likely candidates for involvement in visual analgesia, given the extensive connectivity between posterior parietal and somatosensory cortices (Pandya and Seltzer, 1982). We measured pain ratings of laser stimuli, while participants viewed either their own hand or a neutral object, during fMRI. We identified brain networks activated by painful laser stimulation and by viewing the body. Critically, we further assessed whether effective connectivity between these posterior parietal cortex and regions in the putative pain network varied when seeing the body versus an object.

\section{Materials and Methods}

Participants. Fourteen healthy individuals (3 female) aged 19-44 years participated for payment. All were right-handed as assessed by the Edinburgh Inventory (range: 17.65-100) and had normal or corrected vision by self-report. One additional participant withdrew midway through the study; his data were excluded. All procedures were approved by the local ethics committee.

Laser stimuli. Painful stimuli were delivered by a neodymium yttrium aluminum perovskite (Nd:YAP) laser (EL.EN. Group) with a wavelength of $1.34 \mu \mathrm{m}$, as used in previous MRI studies of laser-induced pain (Watson et al., 2009; Ploner et al., 2011). Stimuli at four different intensities $(1.5,2.0,2.5$, and $3.0 \mathrm{~J} ; 4 \mathrm{~ms}$ pulse duration; $6 \mathrm{~mm}$ beam diameter) were delivered to the dorsum of the right hand. Note that lasers deliver energy, rather than temperature per se.

Procedure. Participants lay supine, looking out of the bore of the scanner via an angled mirror placed directly above the face. The participants' right hand rested on an angled $\left(30-45^{\circ}\right)$ shelf above the torso, so they could have a clear view of their hand. Four green LEDs were placed on the shelf in a square arrangement bounding the hand. A specially constructed box could be placed on top of the hand to occlude it from view while still allowing access for the experimenter to apply laser stimulation. A wooden object of approximately hand size was fixed to the front of the box and when in location could be clearly seen by the participant at approximately the same gaze angle as the (now occluded) hand. Four LEDs were embedded into the corners of the object, so that the LED appearance remained the same whether the object or hand was visible.

The experiment comprised three sessions, each divided into 12 blocks of $46 \mathrm{~s}$, which alternated between vision of the hand or object. The initial condition (i.e., hand or object) was counterbalanced across participants. Within each block, four laser stimuli were delivered, one at each of 1.5, 2, 2.5 , or $3 \mathrm{~J}$, in random order. The time preceding each laser stimulus was either $8,9,10$, or $11 \mathrm{~s}$, in random order. To avoid habituation or sensitization effects, the hand dorsum was divided into rough quadrants, and a laser stimulus was applied to each such quadrant only once within each block, again in random order. An experimenter in the scanner room positioned the stimulator above a pseudorandom point within the specified quadrant, but the timing of the stimulus was controlled by a computer in the control room. When each laser stimulus was delivered, the four LEDs surrounding either the hand or the object flashed briefly. The LED onset served as a cue that a stimulus had been delivered, and their offset served as a cue for the participant to rate pain intensity using a $0-100$ scale ( 0 being no sensation at all, 100 being the worst pain imaginable). Participants responded verbally. Noise cancellation software allowed verbal responses to be recorded in real time and stored for offline confirmation. Blocks were separated by $12 \mathrm{~s}$ of rest in which a sheet of black card was placed in front of the bore of the scanner.

Data acquisition. Data were collected using a 1.5 T Siemens SONATA system. $\mathrm{T}_{2}{ }^{*}$-weighted functional images were acquired using an echoplanar imaging (EPI) sequence. Each EPI consisted of 48 contiguous 2 -mm-thick axial slices ( $1 \mathrm{~mm}$ gap between slices) covering the whole brain $(\mathrm{TR}=4.32 \mathrm{~s}$; $\mathrm{TE}=50 \mathrm{~ms})$. A total of 165 whole-brain volumes were collected in each of three scanning sessions, each lasting $\sim 12 \mathrm{~min}$. The first four volumes of each run were discarded to allow for $\mathrm{T}_{1}$ equil-

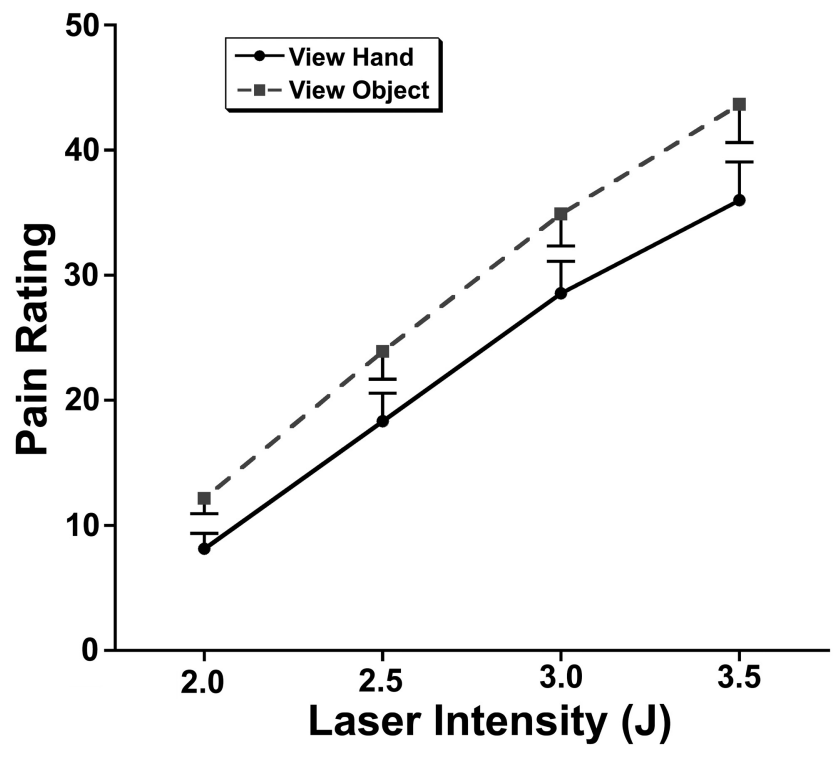

Figure 1. Perceptual results: subjective ratings of pain intensity as a function of stimulation intensity and visual condition. Error bars indicate the SEM difference between the hand and object conditions at each laser intensity.

ibration effects. After the functional sessions, a whole-brain $\mathrm{T}_{1}$-weighted structural scan was acquired, consisting of 176 slices ( $1 \mathrm{~mm}$ isotropic voxel size).

Data analysis. Data were analyzed using Statistical Parametric Mapping (SPM8; Wellcome Centre for Neuroimaging, University College London, London, UK; http://www.fil.ion.ucl.ac.uk/spm). All functional volumes for each participant were realigned to the first volume to correct for head motion. Volumes were then spatially normalized to the Montreal Neurological Institute (MNI) template using DARTEL (Ashburner, 2007), a diffeomorphic image registration algorithm. This has been found to increase intersubject registration compared with independent normalization of data from individual participants (Klein et al., 2009). Structural scans from each participant were first segmented into distinct tissue classes and then registered with each other to produce a template brain reflecting the average of the 14 participants and a "flow field" to allow transformation of each participant's brain to the group average. These flow fields were combined with an affine transformation of the average brain to the MNI template and applied to functional volumes, resulting in spatially normalized data with a voxel size of $2 \times 2 \times 2 \mathrm{~mm}$, and smoothed with an $8 \mathrm{~mm}$ full-width half-maximum Gaussian kernel, in accord with the standard SPM approach.

For each participant, we applied a general linear model (GLM) to each voxel in the functional data using delta (stick) functions to model the eight types of laser stimuli (i.e., 4 intensities by 2 visual conditions, hand or object); plus the onset and offset of the hand or object stimulus. This resulted in 12 stimulus regressors, plus an additional 6 motion parameters, per functional run, which were convolved with a canonical hemodynamic response function. A high-pass filter with a cutoff of $128 \mathrm{~s}$ was also applied to remove low-frequency confounds. Parameter estimates for each regressor were derived and contrast images reflecting each of the 8 laser stimulus regressors were calculated for each participant and entered into a second-level random-effect $4 \times 2$ ANOVA with factors intensity $(1.5,2.0,2.5,3.0 \mathrm{~J})$ and vision (hand, object).

Statistical significance of fMRI activations was determined using a combined voxel and cluster level criterion. Activations were thresholded at $p<0.001$ and a cluster false discovery rate (FDR) of $p<0.05$ was then used to control for multiple comparisons across the brain (Chumbley and Friston, 2009). Because we had a priori hypotheses regarding the well established cortical network responsive to pain (cf. the pain matrix; Tracey and Mantyh, 2007), masks for several areas known to be activated by painful laser stimuli were generated using the Automated Anatomical Labeling toolbox (Tzourio-Mazoyer et al., 2002), including: SI, SII (ro- 


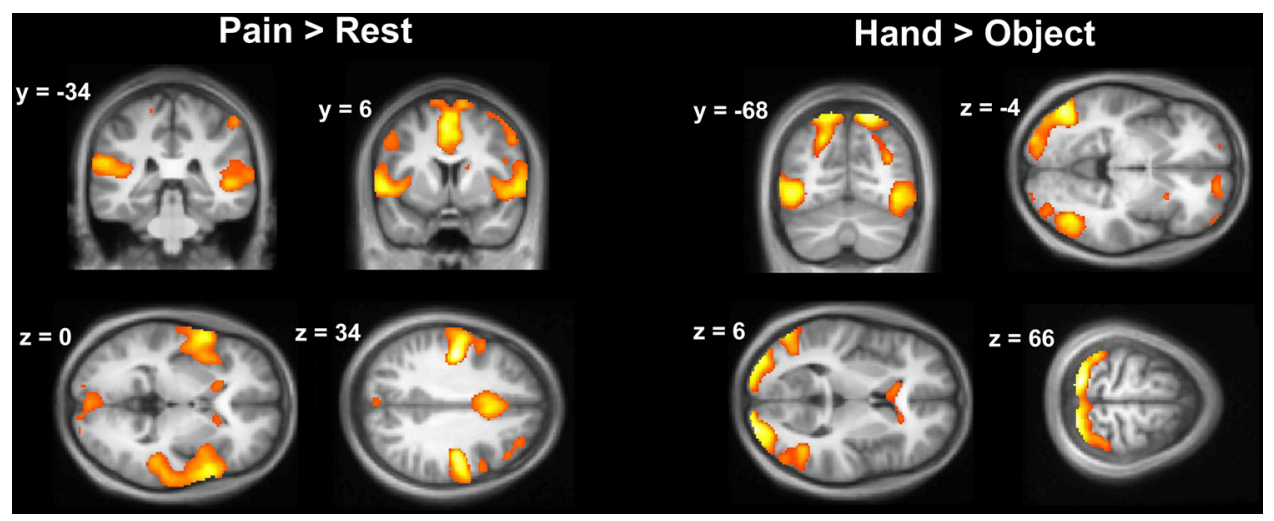

Figure 2. Left, The wide network of areas displaying BOLD fMRI responses to pain-related laser stimulation, including SI, SII, insula, and ACC. Right, fMRI activations to laser stimulation when looking at the hand compared with when looking at the object. An extensive posterior network was activated when laser stimuli were delivered while seeing the body.

Table 1. Areas activated by laser stimulation

\begin{tabular}{|c|c|c|c|c|}
\hline \multirow[b]{2}{*}{ Region } & \multicolumn{3}{|c|}{ MNI coordinates } & \multirow[b]{2}{*}{$t$} \\
\hline & $x$ & $y$ & $z$ & \\
\hline L postcentral gyrus (SI) & -46 & -12 & 36 & 19.38 \\
\hline L postcentral gyrus (SI) & -58 & -6 & 26 & 18.41 \\
\hline R postcentral gyrus (SI) & 58 & -6 & 24 & 17.31 \\
\hline R postcentral gyrus (SI) & 52 & -10 & 42 & 17.20 \\
\hline R precentral gyrus & 44 & -12 & 36 & 16.88 \\
\hline L supplementary motor area & -2 & -2 & 58 & 16.88 \\
\hline L middle cingulate cortex & -4 & 12 & 42 & 15.82 \\
\hline L postcentral gyrus (SI) & -64 & -6 & 14 & 15.71 \\
\hline Ranterior insula & 48 & 12 & -6 & 13.34 \\
\hline L anterior insula & -48 & 10 & -6 & 12.21 \\
\hline L anterior cingulate cortex & -2 & 20 & 32 & 11.88 \\
\hline R anterior cingulate cortex & 2 & 20 & 30 & 11.70 \\
\hline L middle cingulate cortex & 10 & 2 & 72 & 11.23 \\
\hline R supplementary motor area & -2 & -92 & 22 & 10.81 \\
\hline L lingual gyrus & -6 & -82 & -10 & 9.79 \\
\hline R inferior parietal lobule & 54 & -36 & 56 & 9.47 \\
\hline L superior temporal gyrus & -48 & -4 & 0 & 9.41 \\
\hline R precentral gyrus & 18 & -30 & 62 & 8.61 \\
\hline L postcentral gyrus (SI) & -18 & -30 & 62 & 8.61 \\
\hline R precuneus & 12 & -76 & 48 & 8.44 \\
\hline L caudate & -14 & -4 & 16 & 8.42 \\
\hline L rolandic operculum (SII) & -46 & -10 & 8 & 8.42 \\
\hline R middle occipital gyrus & 24 & -98 & 6 & 8.36 \\
\hline L caudate & -16 & 18 & 2 & 8.31 \\
\hline R posterior insula & 34 & -12 & 16 & 6.75 \\
\hline R posterior insula & 46 & -8 & 6 & 6.40 \\
\hline R posterior insula & 42 & -14 & 14 & 5.83 \\
\hline R putamen & 32 & -18 & 6 & 5.30 \\
\hline R posterior insula & 32 & -24 & 12 & 5.18 \\
\hline R posterior insula & 40 & -12 & -6 & 5.10 \\
\hline
\end{tabular}

L, Left; R, right.

landic operculum), insula, anterior cingulate cortex (ACC), and middle cingulate cortex (MCC), bilaterally. Small volume correction was then used for these specific regions of interest (ROIs).

To investigate condition-specific effective connectivity (or functional coupling) between posterior parietal cortex (PPC) within the "visual body network" that we identified (see Results) and the putative pain matrix, we conducted a hypothesis-driven psychophysiological interaction (PPI) analysis (Friston et al., 1997). PPIs assess the extent to which an experimental factor modulates the effective connectivity of one brain region with others, in terms of condition-specific covariation in residuals. Given a specific seed region (here, right and left PPC; see below), PPI identifies voxels which covary differentially with the seed region as a function of an experimental factor. For each participant, first-level GLM analyses were conducted including three regressors: (1) an experimental vector coding whether participants were viewing their hand or the object
Table 2. Comparisons of hand and object

\begin{tabular}{|c|c|c|c|c|}
\hline \multirow[b]{2}{*}{ Region } & \multicolumn{3}{|c|}{ MNI coordinates } & \multirow[b]{2}{*}{$t$} \\
\hline & $x$ & $y$ & $z$ & \\
\hline \multicolumn{5}{|l|}{ Hand $<$ Object } \\
\hline L lingual gyrus & -16 & -74 & -8 & 8.12 \\
\hline R inferior temporal gyrus & 50 & 0 & -40 & 7.18 \\
\hline$R$ cuneus & 8 & -86 & 20 & 5.00 \\
\hline L precentral gyrus & -30 & -28 & 56 & 4.72 \\
\hline R cerebellum & 46 & -66 & -42 & 4.67 \\
\hline R somatosensory cortex & 32 & -20 & 48 & 4.51 \\
\hline L operculoinsular cortex & -42 & -20 & 24 & 4.38 \\
\hline L middle temporal gyrus & -68 & -18 & -6 & 4.26 \\
\hline \multicolumn{5}{|l|}{ Hand $>0$ bject } \\
\hline L superior parietal lobule & -18 & -62 & 66 & 10.26 \\
\hline L middle occipital gyrus & -26 & -84 & 38 & 9.77 \\
\hline R superior occipital gyrus & 24 & -100 & 6 & 9.18 \\
\hline R superior parietal lobule & 16 & -68 & 64 & 9.18 \\
\hline L inferior occipital gyrus & -48 & -72 & -4 & 8.89 \\
\hline R superior occipital gyrus & 28 & -80 & 42 & 8.84 \\
\hline L middle occipital gyrus & -34 & -94 & 10 & 8.39 \\
\hline$R$ cuneus & 16 & -102 & 8 & 8.23 \\
\hline L superior parietal lobule & -16 & -78 & 50 & 8.01 \\
\hline L middle occipital gyrus & -24 & -102 & 6 & 7.82 \\
\hline R inferior temporal gyrus & 44 & -74 & -8 & 7.43 \\
\hline $\mathrm{R}$ middle temporal gyrus & 48 & -64 & -2 & 6.86 \\
\hline R prefrontal cortex & 8 & 60 & -18 & 6.50 \\
\hline R middle occipital gyrus & 38 & -86 & 28 & 6.19 \\
\hline R putamen & 16 & 12 & -10 & 4.88 \\
\hline L caudate & -12 & 22 & 6 & 4.34 \\
\hline L caudate & -4 & 16 & 4 & 4.33 \\
\hline R putamen & 24 & 16 & -6 & 4.20 \\
\hline
\end{tabular}

(the "psychological" regressor), (2) the time-series of activation in the PPC seed region from the visual body network (the "physiological" regressor), and (3) the product of the first two regressors (the PPI regressor), the physiological regressor being deconvolved with a canonical HRF to estimate neural (rather than hemodynamic) activity.

Given the well established connectivity between PPC and somatosensory cortices (Pandya and Seltzer, 1982; Cavada and Goldman-Rakic, 1989), we hypothesized that the PPC nodes of the visual body network would show altered effective connectivity with the putative pain matrix when viewing the body. Separate PPI analyses were, thus, conducted using the PPC peaks in the left and right hemispheres as seeds. To extract time-series of activity, we first determined the group peak voxels for the [Hand $>$ Object] contrast, thresholded at $p<0.05$ corrected for FWE across the whole brain and with a minimum cluster size of 8 voxels, in the left PPC $(-18,-62,66)$ and right PPC $(16,-68,64)$. We then found for each participant the local peak voxel nearest to those group peaks in each hemisphere. We then defined for each PPC the ROI by identifying voxels 


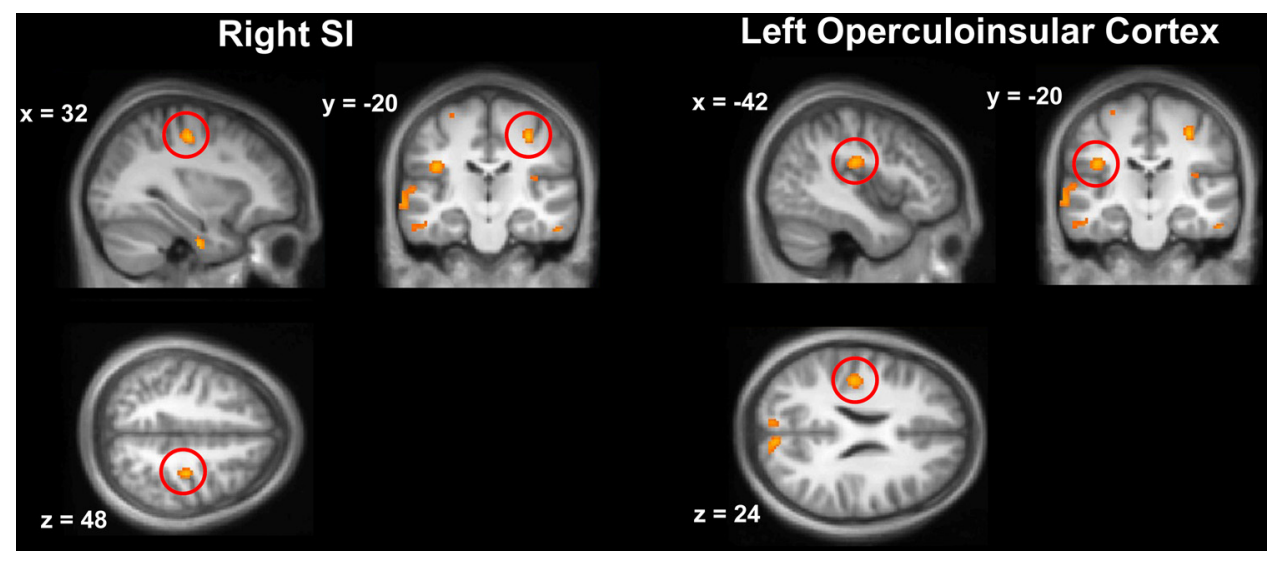

Figure 3. Regions of interest identified from previous studies as coding for experienced pain levels, and here showing reduced activation to laser stimulation when viewing the body in contrast to the object. Left, Right SI. Right, Left operculoinsular cortex.

within a sphere of $6 \mathrm{~mm}$ radius centered on this peak voxel that were active in the [Hand $>$ Object] contrast above a threshold of $p<0.005$ (uncorrected) with a cluster size of at least 4 voxels. The time series for each participant was computed as the first eigenvariate of all voxel time series within the ROI. Contrast estimates for the PPI regressor from each participant were analyzed at the second-level using one-sample $t$ tests.

\section{Results}

Subjective pain ratings

Subjective pain ratings showed a clear monotonic increase with stimulus intensity as expected, $F_{(3,39)}=26.18, p<0.0001$ (Fig. 1). More importantly, pain intensity was significantly reduced when viewing the hand compared with the object (grand means: Hand: 22.8, Object: 28.7$), F_{(1,13)}=10.00, p<0.01$, replicating previous findings that viewing the body is analgesic (Longo et al., 2009; Mancini et al., 2011). Visual context and intensity did not interact, $F_{(3,39)}=0.81$, indicating that vision of the body affects the intercept of the psychometric function, not the slope.

\section{fMRI responses}

Identification of cortical pain network

We first investigated the effect of pain versus rest, and found extensive activations within the set of bilateral brain areas well established to respond to painful stimuli, the so-called pain matrix (Tracey and Mantyh, 2007; Iannetti and Mouraux, 2010). These areas included: SI, SII, anterior insula, posterior insula, ACC, and MCC (Fig. 2, Table 1).

Visual body network: hand > object during laser stimulation

We next investigated the effects of vision of the body on brain responses to laser stimulation. The contrast [Hand $>$ Object] revealed an extensive bilateral network of posterior regions $(\mathrm{Ta}-$ ble 2, Fig. 2), consistent with previous research (for review, see Peelen and Downing, 2007). We refer to this here as the visual body network. This included extensive bilateral activations of the superior parietal lobules, posterior lateral occipital cortex, and occipitotemporal cortex. Additionally, bilateral regions of the basal ganglia were also activated.

\section{Visual analgesia: object $>$ hand during laser stimulation}

Because vision of the body was analgesic, a natural prediction is that areas known to be involved in the cortical processing of pain (as defined by the pain > rest contrast above) might show reduced activation when looking at the hand compared with the object. The contrast [Object $>$ Hand] for the laser stimulation regressor provided some evidence confirming this directional hy- pothesis, within early, sensory regions of the pain matrix. A region of right (ipsilateral) sensorimotor cortex, partially overlapping the SI ROI was identified (32, -20, 48; Fig. 3, Table 2 ), for which activation in the view-object condition was greater than in the view-hand condition during laser stimulation. There was also a cluster straddling the left SII and insula regions $(-42$, $-20,24$; Table 2). Some other brain areas also showed reduced activation when looking at the body (e.g., left medial occipital cortex and right anterior inferior temporal cortex) but were not obviously related to the cortical processing of pain. There was no reduction of activity in the ACC or MCC.

\section{Psychophysiological interactions}

We had hypothesized that the fMRI responses observed during hand observation may subserve visual analgesia in the form of modulated effective connectivity (or functional coupling) with the pain matrix. To test this, we performed a PPI analysis (Friston et al., 1997) using the PPC nodes of the visual body network as seed areas. We focus on PPC seeds because of the well established anatomical connectivity between the posterior parietal cortex and somatosensory cortices (Pandya and Seltzer, 1982; Cavada and Goldman-Rakic, 1989). PPI identifies voxels which show altered functional coupling (residual covariation) with the seed region as a function of psychological context, in this case vision of the hand or the object. We found that vision of the hand led to increased functional coupling between the PPC nodes of the visual body network and bilateral areas of the cortical pain network (Fig. 4, Table 3). These areas notably included SI, SII, the anterior and posterior insula, and the ACC. Thus, the analgesic effect of viewing the body dramatically changed functional coupling of PPC with areas responding to laser stimulation.

\section{Discussion}

Viewing the hand, compared with viewing a neutral object at the same location, reduced the subjective intensity of laser-induced pain and increased functional coupling between posterior parietal nodes sensitive to vision of the body (within the visual body network) and the cortical network traditionally described as the pain matrix. The finding of visually induced analgesia replicates recent results (Longo et al., 2009; Mancini et al., 2011). Interestingly, the behavioral data here further suggest that visual analgesia involves a shift in the intercept of the psychophysical function, rather than its slope. This reduction in intercept for intensity ratings is consistent with the finding of Mancini et al. (2011) that 


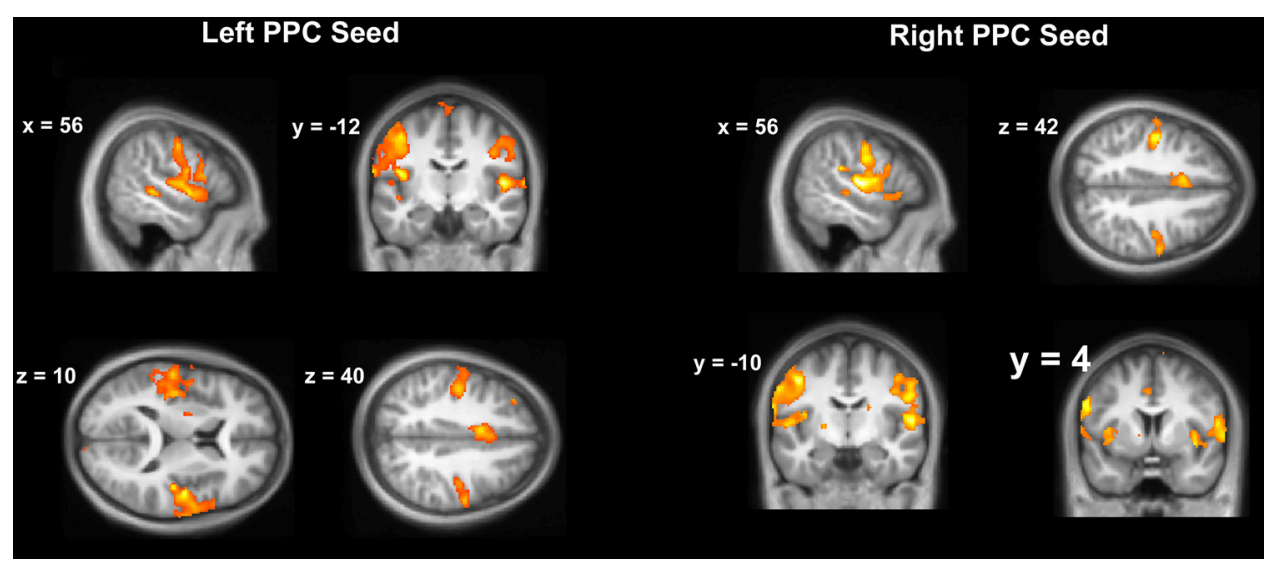

Figure 4. Regions whose functional coupling with PPC nodes in the posterior visual body network increased when viewing the body compared with the object. Vision of the body increased functional coupling between these PPC nodes and regions in the pain network evoked by laser stimulation. Areas overlapping with the pain matrix (i.e., [laser stimulation $>$ rest]) are highlighted, via inclusive masking.

Table 3. Areas showing altered effective connectivity with the left $(-18,-62,66)$ and right $(16,-68,64)$ posterior parietal nodes of the visual body network

\begin{tabular}{|c|c|c|c|c|}
\hline \multirow[b]{2}{*}{ Region } & \multicolumn{3}{|c|}{ MNI coordinates } & \multirow[b]{2}{*}{$t$} \\
\hline & $x$ & $y$ & $z$ & \\
\hline \multicolumn{5}{|l|}{$\begin{array}{l}\text { Left PPC: Increased effective connectivity when } \\
\text { viewing the hand }\end{array}$} \\
\hline L rolandic operculum & -40 & -14 & 16 & 11.79 \\
\hline R parietal operculum & 50 & -12 & 8 & 10.60 \\
\hline R postcentral gyrus & 60 & 0 & 16 & 10.59 \\
\hline L parietal operculum & -68 & -14 & 18 & 9.57 \\
\hline L rolandic operculum & -40 & -34 & 16 & 9.46 \\
\hline R temporal pole/frontal operculum & 54 & 2 & 0 & 8.63 \\
\hline L middle cingulate cortex & -6 & 8 & 38 & 7.93 \\
\hline L middle cingulate cortex & 0 & 14 & 36 & 6.53 \\
\hline RSMA & 10 & 4 & 74 & 6.14 \\
\hline R lingual gyrus & 16 & -100 & -10 & 6.05 \\
\hline \multicolumn{5}{|l|}{$\begin{array}{l}\text { Left PPC: Decreased effective connectivity when } \\
\text { viewing the hand }\end{array}$} \\
\hline L angular gyrus & -48 & -70 & 24 & 6.42 \\
\hline L angular gyrus & -52 & -58 & 32 & 6.28 \\
\hline L middle temporal gyrus & -42 & -58 & 18 & 6.03 \\
\hline \multicolumn{5}{|l|}{$\begin{array}{l}\text { Right PPC: Increased effective connectivity when } \\
\text { viewing the hand }\end{array}$} \\
\hline R superior temporal gyrus/rolandic operculum & 56 & -14 & 8 & 9.76 \\
\hline L precentral gyrus & -60 & 2 & 30 & 9.67 \\
\hline L postcentral gyrus & -64 & -6 & 32 & 9.47 \\
\hline L postcentral gyrus & -44 & -10 & 44 & 9.29 \\
\hline R rolandic operculum & 58 & -2 & 12 & 8.85 \\
\hline R postcentral gyrus & 54 & -10 & 28 & 6.79 \\
\hline R lingual gyrus & 26 & -88 & -6 & 6.41 \\
\hline R inferior occipital gyrus & 22 & -98 & -8 & 6.28 \\
\hline L middle cingulate cortex & -6 & 12 & 42 & 6.01 \\
\hline RSMA & 6 & -2 & 64 & 4.94 \\
\hline \multicolumn{5}{|l|}{$\begin{array}{l}\text { Right PPC: Decreased effective connectivity when } \\
\text { viewing the hand }\end{array}$} \\
\hline L middle temporal gyrus & -48 & -62 & 18 & 6.36 \\
\hline L middle occipital gyrus & -40 & -76 & 32 & 6.06 \\
\hline L angular gyrus & -50 & -70 & 36 & 5.49 \\
\hline
\end{tabular}

$L$, Left; R, right.

viewing the body increases heat-pain thresholds. Further, the absence of any change in slope suggests that vision does not lead to a loss of information about nociceptive inputs, but rather to a reduction in experienced pain levels.

Our fMRI analyses first focused on whether viewing the body reduces activation of key nodes in the so-called pain matrix, since this could provide a potential neural marker of visual analgesia.
Using small volume corrections on sites derived from previous studies, we only found reduced activation in SI and the operculoinsular cortex when viewing the hand during laser stimulation, compared with the neutral object. We note that such reductions were not observed in ACC. This pattern of results suggests that visual analgesia may involve only restricted regions within the larger cortical pain network. Results from visual-tactile experiments (below pain threshold) also indicate that viewing the body can influence processing in early somatosensory cortex. For example, viewing the body modulates tactile acuity (Kennett et al., 2001b), early somatosensory cortical potentials (Longo et al., 2011), and somatosensory intracortical inhibition (Cardini et al., 2011).

Unimodal stimuli can sometimes produce deactivations of primary sensory cortices for other modalities (Lewis et al., 2000; Laurienti et al., 2002; Shulman et al., 1997). Could the reduced activation of pain matrix areas we report reflect merely such cross-modal suppression? We consider this unlikely for several reasons. First, while many studies have reported suppression of visual or auditory cortices following unimodal stimulation in a different modality (Laurienti et al., 2002; Lewis et al., 2000), deactivations of somatosensory cortex following unimodal visual stimuli have typically not been found even in studies specifically testing for them (Shulman et al., 1997; Mouraux et al., 2011).

Second, as described in the introduction, recent findings (Mouraux et al., 2011) have demonstrated that the so-called pain matrix can in fact be positively activated also by unimodal visual stimuli. Accounts based on cross-modal suppression account would have rather predicted deactivations. In fact, patterns of activation nearly identical to the canonical pain matrix can be produced by unimodal visual, auditory, and non-nociceptive somatosensory stimuli (Mouraux et al., 2011), and these activations are positively correlated with subjective salience. There is also evidence that such facilitative relations between visual and somatosensation are reciprocal. Findings by Sathian and colleagues indicate clear activations of visual cortices by unimodal tactile stimuli (Sathian et al., 1997; Sathian and Zangaladze, 2002; Peltier et al., 2007). Similarly, another recent study found increased SI activation following visuo-tactile stimulation compared with touch alone (Dionne et al., 2010). Given such data, mere visual salience of the hand compared with the object in our experiment should, if anything, have increased activation of the pain matrix. 
In contrast, we found reduced pain-related activation in the specific context of visual analgesia.

Third, a number of studies have measured fMRI activations while subjects view hands (Ehrsson et al., 2004; Myers and Sowden, 2008; Tsakiris et al., 2010). These studies typically do not report deactivation of SI or other somatosensory regions following visual perception of bodies. To our knowledge, the only finding of this kind is the report by Saxe et al. (2006) of SI deactivation when viewing body parts presented in an allocentric perspective. Crucially, however, the same study found no deactivation when the hand was presented in a first-person perspective, as in our study (see also Ruby and Decety, 2001). Thus, merely seeing one's own hand (outside the context of visual analgesia) appears to activate SI, rather than deactivate it as we found here for the specific context of visual analgesia.

We found extensive and robust activations when contrasting hand $>$ object during laser stimulation. These activations were bilateral and posterior, and included lateral occipital and superior parietal areas. Our results therefore agree with the proposed roles of these areas in visual representation of the body and peripersonal space (Schlack et al., 2005; Peelen and Downing, 2007; Orlov et al., 2010). The strong bilateral activation of occipitotemporal areas appears consistent with the known responses of the EBA (Downing et al., 2001; Myers and Sowden, 2008; Costantini et al., 2011). We also found strong activation of SPL areas traditionally associated with multisensory representation of peripersonal space (Duhamel et al., 1998; Pitzalis et al., 2010). We note there appears to be little overlap between this visual body network and the classic pain network. However the basal ganglia provides an exception to this: viewing the hand activated the caudate nucleus and putamen, which are also known to be activated by painful laser stimuli (Coghill et al., 1999; Bingel et al., 2004), and which produce decreased sensitivity to heat pain when damaged (Starr et al., 2011). Further, Starr et al. (2011), using probabilistic tractography, found that areas of the putamen activated by painful stimuli have extensive anatomical connectivity with numerous cortical and subcortical regions involved in emotion and memory (amygdala and hippocampus), nociceptive processing (insula, ACC), and higher-order cognition (premotor cortex, BA8). Thus, the basal ganglia might potentially play a key role in modulating pain, via its anatomical connections with limbic, sensorimotor, and cognitive "loops" (Alexander and Crutcher, 1990; Crittenden and Graybiel, 2011).

Some authors have recently suggested that pain may be an emergent property of connectivity within the pain network (Tracey, 2011), rather than a readout of activity at any individual node within the pain network. This view appears strengthened by the apparent absence of any single neural correlate of pain intensity following nociceptive laser stimulation (Iannetti et al. 2008). Moreover, even resting functional connectivity within the pain network can predict the perceived intensity of a subsequent pain stimulus (Ploner et al., 2010). In addition, previous studies have shown that functional connectivity between nociceptive centers and other brain networks can underlie modulation of pain. For example, attentional and emotional modulations of pain involve increased connectivity between the insula and wider brain networks for attention or emotion, respectively (Ploner et al., 2011). But most previous work focused on possible connectivity changes underlying top-down modulation of pain by higher-level cognition, such as attention and expectation (Wiech et al., 2008). Our results extend the notion that pain modulation can involve changing interplay between the putative pain matrix and other brain networks. A distinctive feature of our study is that the key interplay involved multisensory areas for representing the body and peripersonal space, while the key psychological manipulation was simply bottom-up viewing of the hand, rather than topdown expectation.

Laser stimulation is unusual in generating pain in the absence of any apparently damaging stimulus. Could this sensory conflict influence visual analgesia? We previously found that whether or not the participant saw the laser being applied had no effect on visual analgesia (Longo et al., 2009). We further showed that visual analgesia is also obtained when participants see (or have the illusion of seeing) the painful stimulus actually touching their hand (Mancini et al., 2011). The presence of a clearly damaging stimulus may very well generate emotional and expectancy responses which influence the experience of pain, but these are distinct from visual analgesia.

Our results thus suggest a novel mechanism of analgesia based on multisensory interactions involving perception of one's own body. Studies of chronic pain following amputation suggest a strong association between pain levels and the cortical representation of one's own body. For example, phantom limb pain after amputation may be a by-product of reorganization of cortical maps of the body (Flor et al., 2006). Interestingly, some reports suggest that simply viewing the body (or the mirror image of an intact limb appearing where the amputated limb is now missing) can reduce phantom limb pain (Ramachandran et al., 1995), and may do so through signals that control plastic processes of cortical reorganization (Lotze et al., 2001; Diers et al., 2010). That is, integrating the painful body part within a stable representation of the body may be an important mechanism for pain regulation. Our study documents an effect of viewing the body on brain activations for acute, rather than chronic pain. Viewing the body can change functional coupling with the pain network. We show that linking acute pain to the brain's visual body representations has a rapid analgesic effect. These effects appear to operate within a much shorter timescale than neuroplastic reorganization associated with chronic pain modulation. Functional linkage between cortical networks could be an important mechanism for promoting neuroplastic changes that may reduce chronic pain.

\section{References}

Alexander GE, Crutcher MD (1990) Functional architecture of basal ganglia circuits: neural substrates of parallel processing. Trends Neurosci $13: 266-271$.

Ashburner J (2007) A fast diffeomorphic image registration algorithm. Neuroimage 38:95-113.

Bingel U, Gläscher J, Weiller C, Büchel C (2004) Somatotopic representation of nociceptive information in the putamen: an event-related fMRI study. Cereb Cortex 14:1340-1345.

Cardini F, Longo MR, Haggard P (2011) Vision of the body modulates somatosensory intracortical inhibition. Cereb Cortex 21:2014-2022.

Cavada C, Goldman-Rakic PS (1989) Posterior parietal cortex in rhesus monkey: I. Parcellation of areas based on distinctive limbic and sensory corticocortical connections. J Comp Neurol 287:393-421.

Chumbley JR, Friston KJ (2009) False discovery rate revisited: FDR and topological inference using Gaussian random fields. Neuroimage 44:62-70.

Coghill RC, Sang CN, Maisog JM, Iadarola MJ (1999) Pain intensity processing within the human brain: a bilateral, distributed mechanism. J Neurophysiol 82:1934-1943.

Costantini M, Urgesi C, Galati G, Romani GL, Aglioti SM (2011) Haptic perception and body representation in lateral and medial occipitotemporal cortices. Neuropsychologia 49:821-829.

Critchley M (1953) The parietal lobes. London: Edward Arnold and Co.

Crittenden JR, Graybiel AM (2011) Basal ganglia disorders associated with imbalances in the striatal striosome and matrix compartments. Front Neuroanat 5:59. 
Derbyshire SW, Jones AK, Gyulai F, Clark S, Townsend D, Firestone LL (1997) Pain processing during three levels of noxious stimulation produces differential patterns of central activity. Pain 73:431-445.

Diers M, Christmann C, Koeppe C, Ruf M, Flor H (2010) Mirrored, imagined and executed movements differentially activate sensorimotor cortex in amputees with and without phantom limb pain. Pain 149:296-304.

Dionne JK, Meehan SK, Legon W, Staines WR (2010) Crossmodal influences in somatosensory cortex: interaction of vision and touch. Hum Brain Mapp 31:14-25.

Downing PE, Jiang Y, Shuman M, Kanwisher N (2001) A cortical area selective for visual processing of the human body. Science 293:2470-2473.

Duhamel JR, Colby CL, Goldberg ME (1998) Ventral intraparietal area of the macaque: congruent visual and somatic response properties. J Neurophysiol 79:126-136.

Ehrsson HH, Spence C, Passingham RE (2004) That's my hand! Activity in premotor cortex reflects feeling of ownership of a limb. Science 305:875-877.

Flor H, Nikolajsen L, Staehelin Jensen T (2006) Phantom limb pain: a case of maladaptive CNS plasticity? Nat Rev Neurosci 7:873-881.

Friston KJ, Buechel C, Fink GR, Morris J, Rolls E, Dolan RJ (1997) Psychophysiological and modulatory interactions in neuroimaging. Neuroimage 6:218-229.

Graziano MS, Yap GS, Gross CG (1994) Coding of visual space by premotor neurons. Science 266:1054-1057.

Iannetti GD, Mouraux A (2010) From the neuromatrix to the pain matrix (and back). Exp Brain Res 205:1-12.

Iannetti GD, Zambreanu L, Cruccu G, Tracey I (2005a) Operculoinsular cortex encodes pain intensity at the earliest stages of cortical processing as indicated by amplitude of laser-evoked potentials in humans. Neuroscience 131:199-208.

Iannetti GD, Hughes NP, Lee MC, Mouraux A (2008) Determinants of laser-evoked EEG responses: pain perception or stimulus saliency? J Neurophysiol 100:815-828.

Kennett S, Eimer M, Spence C, Driver J (2001a) Tactile-visual links in exogenous spatial attention under different postures: convergent evidence from psychophysics and ERPs. J Cogn Neurosci 13:462-478.

Kennett S, Taylor-Clarke M, Haggard P (2001b) Noninformative vision improves the spatial resolution of touch in humans. Curr Biol 11:1188-1191.

Klein A, Andersson J, Ardekani BA, Ashburner J, Avants B, Chiang MC, Christensen GE, Collins DL, Gee J, Hellier P, Song JH, Jenkinson M, Lepage C, Rueckert D, Thompson P, Vercauteren T, Woods RP, Mann JJ, Parsey RV (2009) Evaluation of 14 nonlinear deformation algorithms applied to human brain MRI registration. Neuroimage 46:786-802.

Laurienti PJ, Burdette JH, Wallace MT, Yen YF, Field AS, Stein BE (2002) Deactivation of sensory-specific cortex by cross-modal stimuli. J Cogn Neurosci 14:420-429.

Lewis JW, Beauchamp MS, DeYoe EA (2000) A comparison of visual and auditory motion processing in human cerebral cortex. Cereb Cortex 10:873-888.

Longo MR, Betti V, Aglioti SM, Haggard P (2009) Visually induced analgesia: seeing the body reduces pain. J Neurosci 29:12125-12130.

Longo MR, Azañón E, Haggard P (2010) More than skin deep: body representation beyond primary somatosensory cortex. Neuropsychologia 48:655-668.

Longo MR, Pernigo S, Haggard P (2011) Vision of the body modulates processing in primary somatosensory cortex. Neurosci Lett 489:159-163.

Lotze M, Flor H, Grodd W, Larbig W, Birbaumer N (2001) Phantom movements and pain: an fMRI study in upper limb amputees. Brain 124:2268-2277.

Mancini F, Longo MR, Kammers MP, Haggard P (2011) Visual distortion of body size modulates pain perception. Psychol Sci 22:325-330.

Mouraux A, Iannetti GD (2009) Nociceptive laser-evoked brain potentials do not reflect nociceptive-specific neural activity. J Neurophysiol 101:3258-3269.

Mouraux A, Diukova A, Lee MC, Wise RG, Iannetti GD (2011) A multisensory investigation of the functional significance of the "pain matrix". Neuroimage 54:2237-2249.

Myers A, Sowden PT (2008) Your hand or mine? The extrastriate body area. Neuroimage 42:1669-1677.

Orlov T, Makin TR, Zohary E (2010) Topographic representation of the human body in the occipitotemporal cortex. Neuron 68:586-600.

Pandya DN, Seltzer B (1982) Intrinsic connections and architectonics of posterior parietal cortex in the rhesus monkey. J Comp Neurol 204:196-210

Peelen MV, Downing PE (2005) Selectivity for the human body in the fusiform gyrus. J Neurophysiol 93:603-608.

Peelen MV, Downing PE (2007) The neural basis of visual body perception. Nat Rev Neurosci 8:636-648.

Peltier S, Stilla R, Mariola E, LaConte S, Hu X, Sathian K (2007) Activity and effective connectivity of parietal and occipital cortical regions during haptic shape perception. Neuropsychologia 45:476-483.

Pitzalis S, Sereno MI, Committeri G, Fattori P, Galati G, Patria F, Galletti C (2010) Human V6: the medial motion area. Cereb Cortex 20:411-424.

Ploner M, Lee MC, Wiech K, Bingel U, Tracey I (2010) Prestimulus functional connectivity determines pain perception in humans. Proc Natl Acad Sci U S A 107:355-360.

Ploner M, Lee MC, Wiech K, Bingel U, Tracey I (2011) Flexible cerebral connectivity patterns subserve contextual modulations of pain. Cereb Cortex 21:719-726.

Ramachandran VS, Rogers-Ramachandran D, Cobb S (1995) Touching the phantom limb. Nature 377:489-490.

Ruby P, Decety J (2001) Effect of subjective perspective taking during simulation of action: a PET investigation of agency. Nat Neurosci 4:546-550.

Sathian K, Zangaladze A (2002) Feeling with the mind's eye: contribution of visual cortex to tactile perception. Behav Brain Res 135:127-132.

Sathian K, Zangaladze A, Hoffman JM, Grafton ST (1997) Feeling with the mind's eye. Neuroreport 8:3877-3881.

Saxe R, Jamal N, Powell L (2006) My body or yours? The effect of visual perspective on cortical body representations. Cereb Cortex 16:178-182.

Schlack A, Sterbing-D’Angelo SJ, Hartung K, Hoffmann KP, Bremmer F (2005) Multisensory space representations in the macaque ventral intraparietal area. J Neurosci 25:4616-4625.

Shulman GL, Corbetta M, Buckner RL, Raichle ME, Fiez JA, Miezin FM, Petersen SE (1997) Top-down modulation of early sensory cortex. Cereb Cortex 7:193-206.

Starr CJ, Sawaki L, Wittenberg GF, Burdette JH, Oshiro Y, Quevedo AS, McHaffie JG, Coghill RC (2011) The contribution of the putamen to sensory aspects of pain: insights from structural connectivity and brain lesions. Brain 134:1987-2004.

Tracey I (2011) Can neuroimaging studies identify pain endophenotypes in humans? Nat Rev Neurol 7:173-181.

Tracey I, Mantyh PW (2007) The cerebral signature for pain perception and its modulation. Neuron 55:377-391.

Tsakiris M, Longo MR, Haggard P (2010) Having a body versus moving your body: neural signatures of agency and body-ownership. Neuropsychologia 48:2740-2749.

Tzourio-Mazoyer N, Landeau B, Papathanassiou D, Crivello F, Etard O, Delcroix N, Mazoyer B, Joliot M (2002) Automated anatomical labeling of activations in SPM using a macroscopic anatomical parcellation of the MNI MRI single-subject brain. Neuroimage 15:273-289.

Watson A, El-Deredy W, Iannetti GD, Lloyd D, Tracey I, Vogt BA, Nadeau V, Jones AK (2009) Placebo conditioning and placebo analgesia modulate a common brain network during pain anticipation and perception. Pain 145:24-30.

Wiech K, Ploner M, Tracey I (2008) Neurocognitive aspects of pain perception. Trends Cogn Sci 12:306-313. 